\title{
Carcinogenesis in ulcerative colitis: Is surveillance worthwhile?
}

\author{
ATR AXON
}

ABSTRACT: Observations that dysplastic epithelium predicts neoplastic change in the colitic bowel have led to the adoption of surveillance policies in ulcerative colitis patients, usually involving colonoscopy irrespective of symptomatology. Few satisfactory prospective studies of surveillance have been carried out. The present data suggest that patients should be screened at 10 years and investigated when symptomatic. Annual clinical review with five-yearly colonoscopic examination may be more reasonable, lead to better compliance, detect the occasional early cancer, and reduce the colonoscopic load. Can J Gastroenterol 1990;4(7):397-399

Key Words: Cancer, Cancer surveillance, Ulcerative colitis

\section{Carcinogenèse et colite ulcéreuse: Surveillance?}

RESUME: Le fait que l'épithélium dysplasique annonce des changements néoplasiques du côlon a entraîné l'adoption de politiques de surveillance visant les patients porteurs de colite ulcéreuse (colonoscopie régulière quelle que soit le symptomatologie). Peu d'études de surveillance prospectives satisfaisantes ont été effectuées. Les données actuelles suggèrent que les patients devraient subir des examens de dépistage à 10 ans et lorsqu'ils présentent des symptômes. Il serait peut-être plus raisonnable de préconiser des examens cliniques annuels accompagnés de côlonoscopie tous les cinq ans; cette approche résulte en effet en une meilleure observance et permet la détection précoce des cancers tout en réduisant le nombre des côlonoscopies.

Gastroenterology Unit, The General Infirmary, Leeds, United Kingdom

Correspondence and reprints: Dr ATR Axon, Gastroenterology Unit, The General Infirmary, Leeds LSI 3EX, United Kingdom
$\mathrm{T}$ HE ASSOCIATION BETWEEN ULcerative colitis and colonic cancer has been recognized for many years (1). In 1967 Morson and Pang (2) showed that the presence of dysplastic epithelium in the rectum was a predictor of neoplastic change elsewhere in the colitic bowel. These observations have led to the adoption of surveillance policies in patients with ulcerative colitis. Those at risk are patients who have had an extensive attack of ulcerative colitis, the period of risk beginning 10 years after the initial attack. Many gastroenterologists now perform regular colonoscopies in this group (3). The presence of either high grade dysplasia or a dysplasia-associated mass or lesion (4) are taken as indications for surgery.

\section{CANCER RISK AND} SURVEILLANCE STUDIES

The cumulative cancer risk in patients with extensive colitis is approximately $7 \%$ at 20 years and $17 \%$ at 30 (5). A number of surveillance 
TABLE 1

Ulcerative colitis surveillance studies

\begin{tabular}{lcccc}
\hline Author (year) & Patients & $\begin{array}{c}\text { Colonoscopies } \\
\text { per patient }\end{array}$ & Excluded or $\geq$ C & Surveillance A/B \\
\hline Blackstone (1981) & 112 & 1.5 & 6 & 0 \\
Lennard-Jones & 119 & 0.92 & 14 & 2 \\
(1983) & 151 & $?$ & 6 & 0 \\
Nugent (1984) & 248 & 1.49 & 7 & 0 \\
Rosenstock (1985) & 71 & 2.1 & 0 & 1 \\
Brostrom (1986) & 112 & 3.3 & 3 & 1 \\
Manning (1987) & 313 & $?$ & 7 & 2 \\
Jones (1988) & 1126 & & 43 & 6 \\
Total & & & & \\
\hline A.B.Cre
\end{tabular}

A.B,C refer to Duke's stages

\section{TABLE 2}

Amalgamated data from uicerative colitis surveillance studies ( 1126 patients)

\begin{tabular}{lccc}
\hline & \multicolumn{3}{c}{ Duke's stage } \\
Circumstance of cancer diagnosis & A & B & $\geq$ C \\
\hline Mass or perforation & & & 2 \\
Barium or sigmoidoscopic diagnosis & 2 & 7 & 7 \\
Colonoscopy performed because of barium appearance & & 4 & 1 \\
Operation for debility & 5 & 4 & 1 \\
Screening colonoscopy & 1 & 1 & 2 \\
Operation for low grade dysplasia & & & 2 \\
Cancer found as a result of surveillance & 5 & 1 & 4 \\
\hline
\end{tabular}

Amalgamated from the studies in Table 1

studies have confirmed an increased incidence of cancer, and have concluded that surveillance may be an effective means of reducing mortality in ulcerative colitis-associated cancer $(4,6,11)$. This review of the literature does not support this view, and if the cost of surveillance, both financial and human, are taken into consideration, their benefit is subject to doubt.

Most studies have been retrospective with incomplete follow-up.

They have included patients referred to tertiary centres with new symptoms and individuals with cancers diagnosed using techniques other than the surveillance method (including those in whom the diagnosis was made at operation for continuous symptoms). In these programs a number of patients had advanced malignant disease at the time of diagnosis.

\section{STUDY DESIGN}

In order to answer the question 'does surveillance work?", the term 'surveillance' must first be defined. Patients newly referred to gastroenterologists with symptomatic ulcerative colitis are often colonoscoped to assess disease activity and extent; others are colonoscoped during their period of illness because of new symptoms or because they have been referred from other centres; and some are colonoscoped who surface after many years of not being followed up. These patients are often assessed or screened by colonoscopy, but this does not constitute 'surveillance'. Surveillance is an ongoing commitment to a program in which patients are followed up irrespective of symptomatology with a view to early detection of a malignant or premalignant lesion. Studies should be prospective and the surveillance technique to be tested agreed to at the outset.

There must be a policy agreed to on the management of dysplasia. In a number of studies, patients appear to have been operated on at random, sometimes with dysplasia being taken into account; at other times it is ignored.

There must be an agreed to endpoint which should include some definition of success or failure. For example the discovery of a Duke's stage C lesion probably represents failure of the pro- gram, and an A or B success only if it is identified and treated by following the surveillance protocol.

Finally, the study should be carried out on an 'intention to survey' basis analogous to the 'intention to treat' protocols used in drug trials. This means that patients who have defaulted from the trial and then develop cancer must be counted as failures. This latter point is of some importance because authors have drawn attention to an apparently increased incidence of cancer in defaulters; this could be construed as evidence in favour of surveillance on the grounds that had they been properly followed up their neoplastic disease would have been diag. nosed earlier. In fact it represents failure of the surveillance program.

\section{PUBLISHED SURVEILLANCE SERIES}

Published series are listed in Table 1 . It can be seen that the average number of colonoscopies per patient ranges from 0.92 to 3.3. If it is accepted that surveillance implies regular annual colonoscopy, and that the initial colonoscopy is screening not surveillance, then few of these centres have in practice instituted a surveillance program.

Table 2 shows the amalgamated data separating colonic cancer into Duke's stages and the circumstances by which cancer was diagnosed: $39 \%$ had Duke's stage Cor worse, while $35 \%$ and $26 \%$ had stages B and A, respectively. These figures underline the inadequacy of current techniques to diagnose cancer in patients known to be at risk, who were seen in first class gastroenterology units where modern investigational techniques were available.

Only $24 \%$ of carcinomas were de. tected as a result of the surveillance program itself, assuming colonoscopy to be the accepted means of surveillance, and only six of 49 cancers $(12 \%)$ were both early (A or B) and dis. covered by surveillance.

\section{POLICY ON MANAGEMENT OF DYSPLASIA}

The management of dysplasia remains controversial and is made more difficult by the interpretive problems 


\section{TABLE 3}

Outcome of 66 patients with dysplasia in ulcerative colitis

\begin{tabular}{lrc}
\hline \multicolumn{2}{l}{$\begin{array}{l}\text { Degree of dysplasia } \\
\text { Severe (17) }\end{array}$} & $\begin{array}{c}\text { Number with } \\
\text { carcinoma }\end{array}$ \\
$\begin{array}{l}\text { Operation } \\
\text { No operation }\end{array}$ & 13 & 5 \\
Moderate (27) & 4 & 0 \\
Operation & 8 & 4 \\
No operation & 19 & 0 \\
Mild (22) & & \\
Operation & 7 & 1 \\
No operation & 15 & 0 \\
\hline
\end{tabular}

Data from reference 6

that less experienced pathologists may have. Table 3, taken from the St Mark's study (6), shows that fewer than half of the patients with dysplasia were operated on (not all of those had severe dysplasia). It might be argued that patients with severe dysplasia but no cancer may have been saved from developing cancer, but this cannot be confirmed without including a control group within the surveillance study. Few gastroenterologists would consider it ethical not to operate on patients with high grade dysplasia, so it is unlikely that this question will be resolved.

Most gastroenterologists would now agree that the finding of mild or moderate (low grade) dysplasia is not a

\section{REFERENCES}

1. Crohn BB, Rosenberg H. The sigmoidoscopic picture of chronic ulcerative colitis (non-specific). Am J Med Sci 1925;170:220-8.

2. Morson BC, Pang LSC. Rectal biopsy as an aid to cancer control in ulcerative colitis. Gut 1967;8:423-34.

3. Lennard-Jones JE. Compliance, cost, and common sense limit cancer control in colitis. Gut 1986;27:1403-7.

4. Blackstone MO, Riddell R, Rogers BHG, Levin B. Dysplasia-associated lesion or mass (DALM) detected by colonoscopy in long-standing ulcerative colitis: An indication for colectomy. reason in itself for surgery because the incidence of cancer in this group is low. In the majority of studies colectomy has been advised only on the basis of high grade dysplasia, a focal lesion associated with dysplasia or cancer itself. In some cases patients were operated for low grade dysplasia. In some it may have been because of the length of history, in others symptoms, in others, possibly, clinical acumen. If, however, low grade dysplasia is not regarded as a reason for universal total colectomy patients found to have cancer as a result of operations for low grade dysplasia should not be regarded as successes of a surveillance program. If this is done it can be seen that only $20 \%$ of cancers were found as a direct result of surveillance. Even this is probably an overestimate because it has been assumed that in all 10 patients colectomy was performed as a result of the surveillance procedure alone rather than debility; this was not specifically stated in all patients.

\section{'COST' OF SURVEILLANCE}

Surveillance programs are difficult to operate. Patients do not like colonoscopy, especially on a regular basis. The unpleasantness and discomfort is to some less daunting than the perceived loss of dignity. To be in a

Gastroenterology 1981;80:366-74.

5. Gyde SN, Prior P, Allan RN, et al. Colorectal cancer in ulcerative colitis: A cohort study of primary referrals from three centres. Gut 1988;29:206-17.

6. Lennard-Jones JE, Ritchie JK, Morson BC, Williams CB. Cancer surveillance in ulcerative colitis - Experience over 15 years. Lancet 1983;ii:149-52.

7. Nugent FW, Haggitt RC. Results of a longterm prospective surveillance program for dysplasia in ulcerative colitis. Gastroenterology 1984;86:1197.

8. Rosenstock E, Farmer RG, Petras R, Sivak MV Jr, Rankin GB, Sullivan $\mathrm{BH}$. Surveillance for colonic carci- regular surveillance program may be reassuring to some, but to others it is a constant reminder that they harbour a premalignant lesion. These considerations lead to a high level of defaulting.

Finally, the financial costs of surveillance studies must be taken into consideration. According to the available data it appears that the cost effectiveness of surveillance programs in ulcerative colitis is low.

\section{CONCLUSIONS}

There is at present no answer to the question "Is surveillance in ulcerative colitis worthwhile?" In spite of evidence that extensive ulcerative colitis carries an increased risk of carcinogenesis, there have been few satisfactory prospective studies. What data there are suggest that patients should be screened at 10 years and investigated when symptomatic. Effective regular annual screening by colonoscopy is not being undertaken in many centres, and where it is, few early cancers are detected. It may be more reasonable to consider annual clinical review with perhaps five-yearly colonoscopic examination. This policy might lead to better compliance, detect occasional early cancers, and reduce the colonoscopic load.

noma in ulcerative colitis.

Gastroenterology 1985;89:1342-6.

9. Broström O, Löfberg R, Öst A,

Reichard H. Cancer surveillance of patients with longstanding ulcerative colitis: A clinical, endoscopical, and histological study. Gut 1986;27:140813.

10. Manning AM, Bulgim OR, Dixon MF, Axon ATR. Screening by colonoscopy for colonic epithelial dysplasia in inflammatory bowel disease. Gut 1987;28:1489-94.

11. Jones HW, Grogono J, Hoare AM. Surveillance in ulcerative colitis: Burdens and benefit. Gut 1988;29:325-31. 


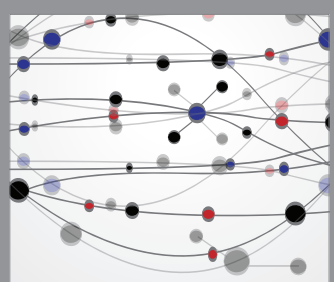

The Scientific World Journal
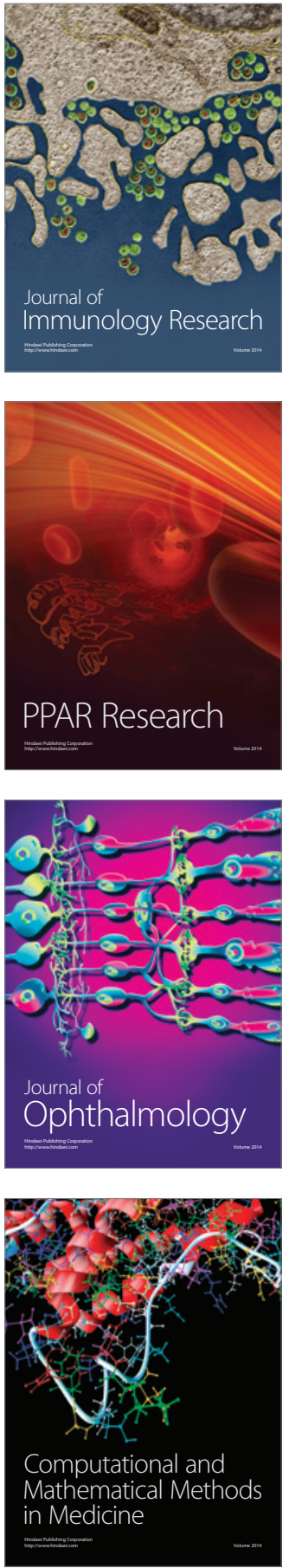

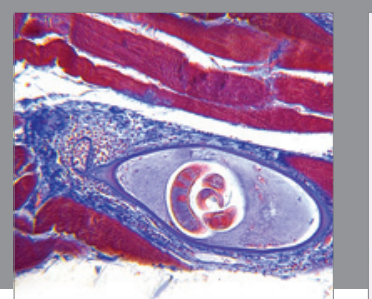

Gastroenterology Research and Practice

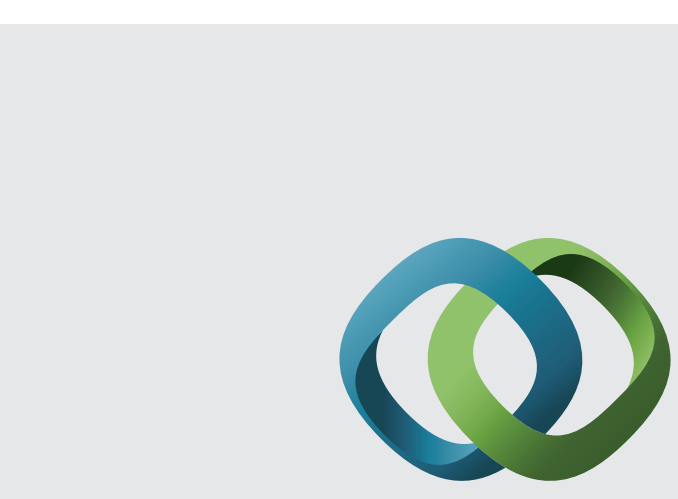

\section{Hindawi}

Submit your manuscripts at

http://www.hindawi.com
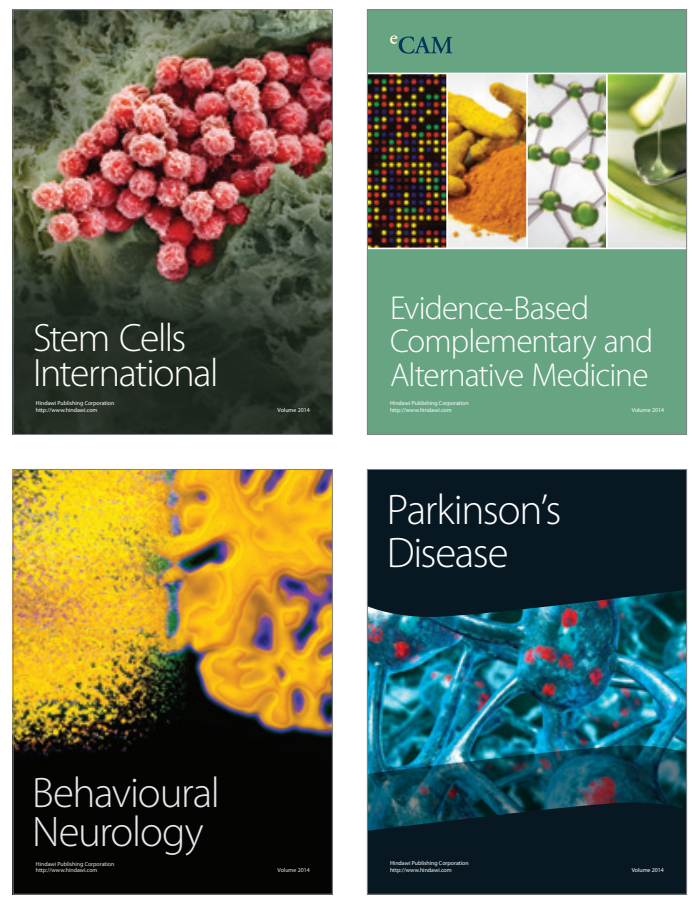
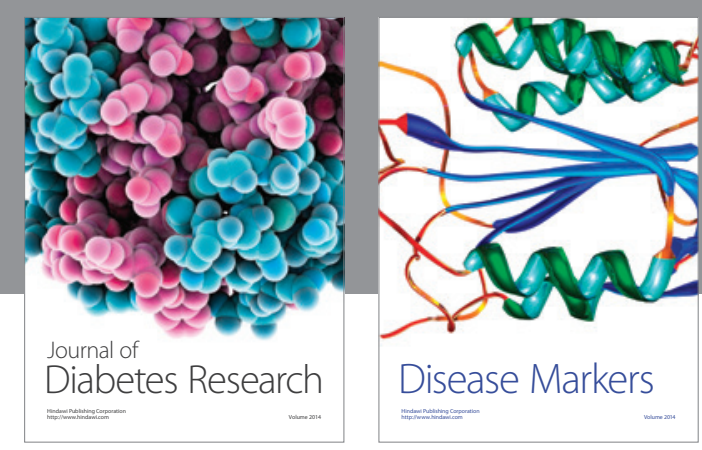

Disease Markers
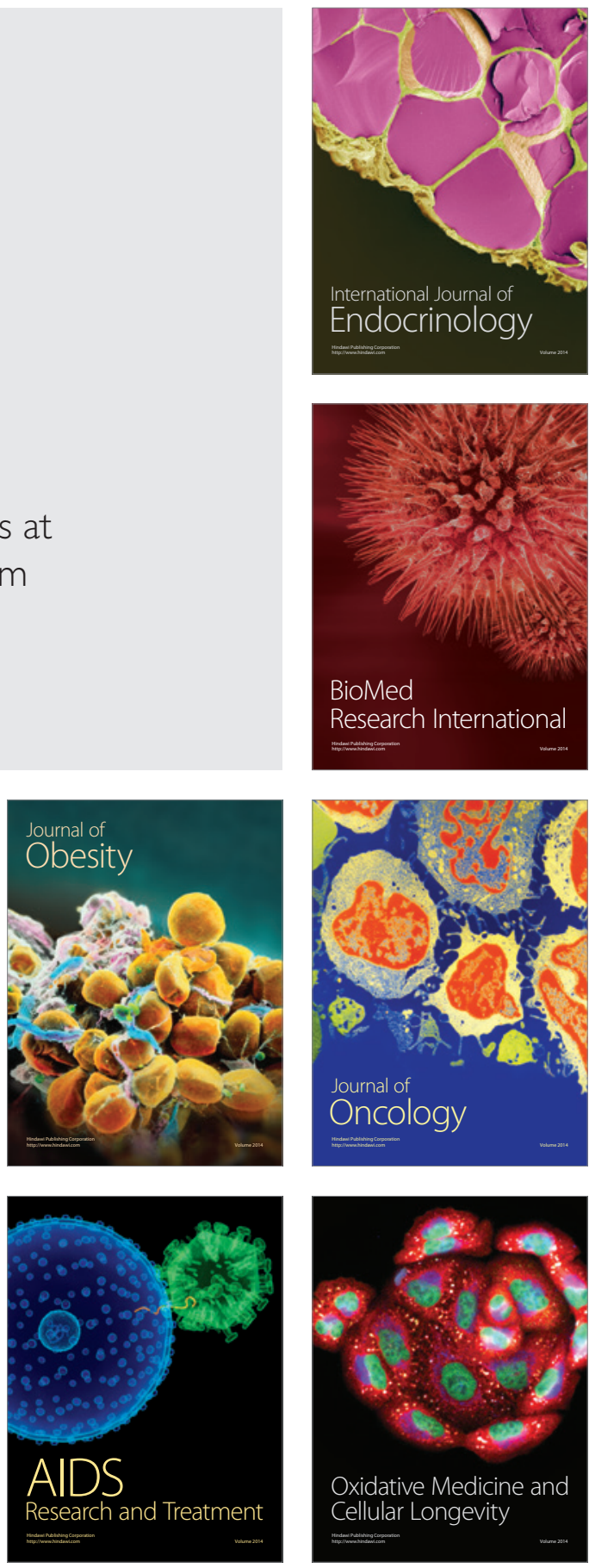